\title{
DEMANDAS JUDICIAIS DE MEDICAMENTOS EM UM MUNICÍPIO POLO DE MINAS GERAIS
}

\author{
LAWSUITS OF MEDICINES ON A POLE MUNICIPALITY OF MINAS GERAIS
}

\author{
Alice Kappel Roque Muncka, Evandro de Oliveira Lupatini ${ }^{b}$, \\ Rouzeli Maria Coelho Pereirac, Rita de Cássia Padula Alves Vieira ${ }^{\mathrm{d}}$ \\ akappelroque@yahoo.com.br, bevandrolupatini@gmail.com, crozelicoelho@uol.com.br, dpadularc@gmail.com \\ Universidade Federal de Juiz de Fora - Juiz de Fora (MG), Brasil
}

Data de recebimento do artigo: 28/01/2016 Data de aceite do artigo: 18/05/2016

\section{RESUMO}

Introduçáo: O Sistema Único de Saúde é o meio pelo qual o Estado brasileiro se propõe a garantir a todos os cidadãos o direito à saúde e à assistência farmacêutica. Contudo, a reclamação por medicamentos na justiça tornou-se um fenômeno expressivo, atingindo de forma notável os municípios, ente federativo mais próximo dos cidadãos. Objetivo: Estudar as açóes judiciais em um município polo da Zona da Mata de Minas Gerais. Materiais e Métodos: Análise documental e retrospectiva das açôes judiciais com pedidos de medicamentos impetradas contra o município de Juiz de Fora, em 2012 e 2013. Resultados: Foram analisados 666 processos judiciais deferidos com pedidos de medicamentos impetrados contra o município, em 2012 e 2013. Observou-se que Juiz de Fora foi o município de domicílio do autor da ação em $57,7 \%$ dos casos. Verificou-se que $62,5 \%$ das prescrições que respaldaram a ação eram provenientes de estabelecimentos públicos de saúde, $70,9 \%$ dos medicamentos demandados náo faziam parte do elenco de nenhum componente de financiamento da assistência farmacêutica, e $85 \%$ dos 20 medicamentos mais pleiteados no período de estudo foram demandados para indicaçôes terapêuticas diferentes das indicaçôes reconhecidas em bula. Conclusóes: Por ser um polo regional de saúde, o município de Juiz de Fora é impelido a estender seu atendimento a populaçóes fora do planejamento original. Torna-se imprescindível investigar as particularidades das demandas judiciais por medicamentos em municípios polos a fim de que o fenômeno da judicialização seja considerado no planejamento da saúde, na articulação interfederativa e na assistência à saúde.

Palavras-chave: Assistência farmacêutica; direito à saúde; medicamento.

\section{ABSTRACT}

Introduction: The Unified Health System is the medium through which the Brazilian State intends to guarantee all citizens the right to health and pharmaceutical care. However, the claim for medicines in courts has become a noteworthy phenomenon in Brazil, reaching in a significant way the municipalities, the federative entities closest to the citizens. Objective: To study the lawsuits in a pole city of Minas Gerais State. Materials and methods: Documentary and retrospective analysis of the lawsuits filed with drug claims against the city of Juiz de Fora, in 2012 and 2013. Results: The analysis was concentrated on 666 deferred lawsuits with medicine requests against the municipality during 2012 and 2013. It was observed that Juiz de Fora was the municipality of the plaintiff's domicile in $57.7 \%$ of the cases. It was found that $62.5 \%$ of prescriptions that supported the action came from public health care establishments, $70.9 \%$ of the medicines were not part of the cast of any pharmaceutical assistance financing component, and $85 \%$ of the 20 most demanded medicines throughout the study were demanded for different therapeutic indications than those of the label directions. Conclusions: Being a regional center for healthcare, Juiz de Fora is driven to extend its services to populations outside the original planning. It is essential to investigate the particularities of judicial demands for medicines in pole cities to ensure that the legalization phenomenon is considered in the planning of healthcare, in the coordination between federal entities, and in healthcare.

Keywords: Pharmaceutical services; right to healthcare; medicines. 


\section{Introdução}

No Brasil, o Sistema Único de Saúde (SUS) é o meio pelo qual o Estado se propóe a garantir a todos os cidadãos o direito aos serviços de atençáo à saúde, abrangendo também a formulaçáo de políticas de medicamentos e a execução de açóes de assistência farmacêutica (AF) que permitam o acesso a medicamentos ${ }^{1}$.

Uma das estratégias da organização das ações de AF visando à eficácia do sistema de distribuição e à promoçâo do uso racional de medicamentos foi sua descentralização para estados e municípios, a fim de reduzir as desigualdades regionais no país e de obter resultados concretos em termos de acesso aos medicamentos ${ }^{2}$.

No entanto, a reclamaçáo por medicamentos na justiça tornou-se um fenômeno expressivo no Brasil, atingindo de forma notável os municípios - ente federativo mais próximo dos cidadãos -, interferindo nas questôes orçamentárias e administrativas relativas à saúde municipal devido ao dever de cumprir a determinação judicial ${ }^{3}$.

Há na literatura diversos estudos sobre as questóes pertinentes ao fornecimento de medicamentos pela via judicial, sobretudo nas capitais brasileiras, perdurando, contudo, a necessidade de avaliação e monitoramento das características relacionadas aos municípios polos, cujo estudo torna-se ainda mais importante, considerando sua relevância na organização do SUS, no planejamento da saúde, na assistência à saúde e na articulação interfederativa, visando à garantia da integralidade da assistência aos usuários ${ }^{4,5}$.

Assim, este estudo objetiva analisar as açôes judiciais que reivindicaram medicamentos em 2012 e 2013 impetradas contra a prefeitura de Juiz de Fora, município polo da Zona da Mata de Minas Gerais, de forma a analisar a relaçâo entre as prescriçôes médicas que respaldaram a decisão judicial, os medicamentos demandados e o seu fornecimento por meio de programas de AF no âmbito do SUS.

\section{Metodologia}

O estudo consistiu em uma análise documental e retrospectiva das açóes judiciais com pedidos de medicamentos impetradas contra o município de Juiz de Fora - município que figura entre os quatro mais populosos de Minas Gerais, sendo um dos 40 municípios brasileiros com mais de 500 mil habitantes e o principal núcleo polarizador da Zona da Mata Mineira e do interior fluminense em termos de saúde, educação e serviços ${ }^{6}$.

Foram incluídos na pesquisa os processos judiciais deferidos, com pedido de pelo menos um medicamento, impetrados contra a Prefeitura Municipal de Juiz de Fora, Minas Gerais, em 2012 e 2013. Foram excluídos os processos deferidos antes de 2012 e depois de 2013 e com pedidos de apenas produtos para a saúde. Os dados foram provenientes das cópias dos processos judiciais e seus anexos arquivados no Setor de Demandas Especiais, responsável pelo cumprimento das açóes judiciais relacionadas à saúde, vinculado à Subsecretaria da Execução Instrumental da Secretaria Municipal de Saúde de Juiz de Fora.

Entre novembro de 2013 e março de 2014, foram consultadas todas as 2.692 pastas contendo processos judiciais arquivadas no Setor de Demandas Especiais. Destas, 130 não foram encontradas, 175 não continham cópia dos autos do processo ou ela estava incompleta, impossibilitando a coleta dos dados, e sete pastas continham um processo em duplicidade, já tendo sido identificado em pastas anteriormente consultadas. Assim, as 2.380 pastas restantes arquivavam um total de 2.388 processos, dos quais 1.722 não atendiam aos critérios de inclusão da pesquisa, sendo, então, analisados 666 processos.

Foram coletados os seguintes dados: número do processo, data de recebimento do processo na Assessoria Jurídica da Secretaria de Saúde, município de domicílio do autor da ação judicial, estabelecimento de saúde que deu origem à prescrição, nome do prescritor responsável pela prescrição que originou o processo, representante do autor da ação, concessão de liminar, ente federativo réu da ação, diagnóstico e medicamento.

Realizou-se o cálculo das proporçóes de açóes por município de domicílio do autor da ação; de ações por representação do autor da ação; de concessão da liminar ou antecipação de tutela; de ações judiciais impetradas por ente federativo réu da ação; de estabelecimento de saúde de origem da prescrição conforme o Cadastro Nacional de Estabelecimentos de Saúde; de especialidade do prescritor - conforme Resolução CFM no 2.005, de 8 de novembro de 2012; de diagnóstico principal - segundo a $10^{\mathrm{a}}$ revisão do Código Internacional de Doença (CID-10); de medicamentos por substância química pelo sistema de classificação anatômico terapêutico e químico (ATC); e de medicamentos que figuram nas relaçóes oficiais de medicamentos por componente do bloco de financiamento da AF, de acordo com o Manual de Indicadores de Avaliação e Monitoramento das Demandas Judiciais de Medicamentos ${ }^{8}$.

Para os medicamentos não contemplados no bloco de financiamento da $\mathrm{AF}$, entre os vinte mais pleiteados, foi verificada a disponibilidade de alternativa terapêutica nas listas de financiamento público do SUS. Consideraram-se, como alternativa terapêutica, os medicamentos de mesmo subgrupo farmacológico do sistema de classificação ATC, para a mesma indicação terapêutica'. Para os medicamentos que não apresentaram alternativa terapêutica disponível no SUS foi verificada a força de recomendação e o grau de evidência para as indicaçóes terapêuticas constantes na bula. 
Para os vinte medicamentos mais demandados no período de estudo, foi verificada a existência de indicaçóes off label, ou seja, medicamentos prescritos para uma indicação terapêutica diferente daquela que foi autorizada pelo órgão regulador brasileiro.

Este estudo foi aprovado pelo Comitê de Ética em Pesquisa Humana da Universidade Federal de Juiz de Fora sob o parecer $n^{\circ} 622.505$.

\section{Resultados}

Foram analisados 666 processos, sendo 318 de 2012 e 348 de 2013. Juiz de Fora foi o município de domicílio do autor da açáo em $57,7 \%$ dos casos analisados. Municípios da microrregião de saúde de Juiz de Fora (Chácara, Matias Barbosa e Piau) e da macrorregiāo de saúde de Juiz de Fora (Bicas e São João Nepomuceno) também foram citados nos autos de processos como domicílios dos autores das açóes (Tabela 1).

Tabela 1: Número de processos judiciais impetrados contra o município de Juiz de Fora por município de domicílio do autor da ação, 2012 e 2013.

\begin{tabular}{|ccc|}
\hline Município de domicílio do autor da açáo & N & $\%$ \\
\hline Juiz de Fora & 384 & 57,7 \\
\hline Bicas & 1 & 0,2 \\
\hline Chácara & 1 & 0,2 \\
\hline Matias Barbosa & 1 & 0,2 \\
\hline Piau & 1 & 0,2 \\
\hline São João Nepomuceno & 1 & 0,2 \\
\hline Sem informação & 277 & 41,6 \\
\hline
\end{tabular}

Fonte: Elaboração própria.

Em todos os processos analisados foi concedida liminar, sendo que 238 deles $(35,7 \%)$ foram referentes a causas com valor de até 20 salários mínimos, sendo o próprio demandante o autor da ação, não requerendo a contratação de advogado para solicitar o medicamento pretendido no Juizado Especial Cível do Tribunal de Justiça de Minas Gerais. A Defensoria Pública do Estado de Minas Gerais foi a representante do autor da ação em 205 (30,8\%) processos, seguida de advogados particulares (149-22,4\%), Defensoria Pública da União (58 - 8,7\%), Ministério Público do Estado de Minas Gerais (13 - 2\%) e Escritório Escola $(2-0,3 \%)$.

O município de Juiz de Fora foi o único ente federativo réu da ação em $543(81,5 \%)$ processos. A União e o estado de Minas Gerais figuraram como réu da ação com o município polo da Zona da Mata mineira em $118(17,7 \%)$ açóes (Tabela 2).
Tabela 2: Entes federativos réus das ações judiciais impetradas contra o município de Juiz de Fora, 2012 e 2013.

\begin{tabular}{|ccc|}
\hline Ente federativo réu da ação & N & \% \\
\hline Município de Juiz de Fora & 543 & 81,5 \\
\hline União, estado e município de Juiz de Fora & 118 & 17,7 \\
\hline Estado e município de Juiz de Fora & 1 & 0,2 \\
\hline União e município de Juiz de Fora & 1 & 0,2 \\
\hline União, estado, município de Bicas & 1 & 0,2 \\
\hline União, estado, município de Bicas e & 1 & 0,2 \\
\hline UNACON ${ }^{1}$ & 1 & 0,2 \\
\hline Uniāo, estado, município e UNACON1
\end{tabular}

Fonte: Elaboração própria.

${ }^{1}$ Unidade de Assistência de Alta Complexidade em Oncologia.

Dos 666 processos, $583(87,5 \%)$ apresentavam pelo menos uma prescrição médica anexada aos autos. Das 616 prescrições analisadas, 451 possibilitaram a consulta de informaçóes sobre o estabelecimento de saúde de origem na base operacional do Cadastro Nacional de Estabelecimentos de Saúde. As prescriçóes que originaram o processo judicial nos anos de 2012 e 2013, em Juiz de Fora, foram provenientes de serviços públicos de saúde em 54,3\% dos casos, sendo que desses a esfera federal foi responsável por $116(25,7 \%)$ prescriçōes e a esfera municipal por 110 (24,4\%). Das 451 prescriçóes que possibilitaram a consulta na base de dados do CNES, $116(25,7 \%)$ foram provenientes de hospitais universitários e $70(15,5 \%)$ de hospitais gerais sem fins lucrativos.

Em 14 prescrições, das 616 analisadas, a identificação do prescritor (nome, assinatura e número de registro no Conselho Regional de Medicina) estava ilegível. Além disso, não constava a identificaçáo impressa da clínica e/ou do profissional. Assim, a ilegibilidade impossibilitou a coleta de informaçóes necessárias para identificação da especialidade do prescritor. As 602 prescrições cujos dados do prescritor estavam legíveis foram prescritas por 287 médicos, sendo que apenas três (1\%) prescritores (um oftalmologista e dois cancerologistas) foram responsáveis por $91(15,1 \%)$ das prescriçóes, enquanto $179(62,4 \%)$ profissionais médicos foram responsáveis por apenas uma prescrição cada.

Os diagnósticos principais por capítulo do CID-10 mais prevalentes foram as neoplasias (19,0\%), seguidas das doenças do olho e anexos (16,6\%) e transtornos mentais e comportamentais $(11,8 \%)$, sendo juntos responsáveis por quase metade $(47,4 \%)$ de todos os diagnósticos analisados.

No biênio estudado, foram demandados 1.013 medicamentos (458 em 2012 e 555 em 2013), relativos a 287 fármacos diferentes, em 666 processos analisados, com média de 1,52 medicamentos por processo. Dos 1.013 medicamentos pleiteados, apenas um - extrato de Vitis vinifera 
L., antioxidante indicado para o auxílio no tratamento de dislipidemias - não foi classificado pelo sistema ATC, pelo fato de o sistema de classificação não contemplar medicamentos fitoterápicos. Dos 1.012 medicamentos classificados, observou-se que $67,7 \%$ dos medicamentos classificados pelo sistema ATC pertenciam a somente quatro grupos anatômicos, assim distribuídos: $276(27,3 \%)$ ao grupo anatômico Sistema Nervoso Central, 155 (15,3\%) ao grupo aparelho digestivo e metabolismo, $148(14,6 \%)$ ao grupo de antineoplásicos e imunomoduladores e 106 $(10,5 \%)$ ao grupo órgãos sensoriais.

Analisando o $5^{\circ}$ nível de classificação do sistema ATC, substância química, pode-se verificar que o ranibizumabe foi o medicamento mais solicitado, seguido do metilfenidato, oxcarbazepina, ácido valproico, bevacizumabe, e brometo de tiotrópio (Tabela 3).

Tabela 3: Número de medicamentos demandados, classificados por substância química (5º́nel ATC), Juiz de Fora, 2012 e 2013.

\begin{tabular}{|c|c|c|c|}
\hline Código ATC & Substância química & $\mathbf{N}$ & $\%$ \\
\hline S01LA04 & Ranibizumabe & 96 & 9,5 \\
\hline N06BA04 & Metilfenidato & 21 & 2,1 \\
\hline N03AF02 & Oxcarbazepina & 19 & 1,9 \\
\hline N03AG01 & Ácido valproico & 18 & 1,8 \\
\hline L01XC07 & Bevacizumabe & 17 & 1,7 \\
\hline R03BB04 & Brometo de tiotrópio & 17 & 1,7 \\
\hline C07AG02 & Carvedilol & 15 & 1,5 \\
\hline L01XC03 & Trastuzumabe & 15 & 1,5 \\
\hline N06AX16 & Venlafaxina & 15 & 1,5 \\
\hline L01XC06 & Cetuximabe & 14 & 1,4 \\
\hline N03AX16 & Pregabalina & 14 & 1,4 \\
\hline L01XC02 & Rituximabe & 13 & 1,3 \\
\hline A03FA03 & Domperidona & 11 & 1,1 \\
\hline A05AA02 & Ácido ursodeoxicólico & 11 & 1,1 \\
\hline A10AB04 & Insulina lispro & 11 & 1,1 \\
\hline B01AC04 & Clopidogrel & 11 & 1,1 \\
\hline L01AX03 & Temozolomida & 11 & 1,1 \\
\hline L01XE05 & Sorafenibe & 11 & 1,1 \\
\hline N05AH04 & Quetiapina & 11 & 1,1 \\
\hline \multirow[t]{3}{*}{ B01AF01 } & Rivaroxabana & 10 & 1,0 \\
\hline & Outros & 651 & 64,3 \\
\hline & Total & 1.012 & 100 \\
\hline
\end{tabular}

Fonte: Elaboração própria.
Do total de medicamentos demandados, 7 (0,7\%) não possuíam registro na Agência Nacional de Vigilância Sanitária (Anvisa): bendamustina $100 \mathrm{mg}$ frasco, captopril $1 \mathrm{mg} / \mathrm{mL}$ solução, captopril $2 \mathrm{mg} / \mathrm{mL}$ solução, captopril $10 \mathrm{mg} / \mathrm{mL}$ solução, espironolactona $1 \mathrm{mg} / \mathrm{mL}$ solução, espironolactona $10 \mathrm{mg} / \mathrm{mL}$ solução, levetiracetam 250 mg comprimido.

Excluindo os 148 (14,6\%) antineoplásicos demandados, que dizem respeito ao bloco de financiamento da Atençáo Especializada de Média e Alta Complexidade, $718(70,9 \%)$ medicamentos náo faziam parte do elenco de nenhum componente de financiamento da AF. Dos medicamentos restantes, 99 (9,8\%) pertenciam ao elenco do Componente Básico, sete $(0,7 \%)$ ao do Componente Estratégico e $41(4,0 \%)$ ao do Componente Especializado, sendo que 106 (10,5\%) medicamentos constam no elenco do Componente Estratégico, mas o CID apresentado no laudo médico ou na prescrição não correspondia à indicação terapêutica contemplada nos Protocolos Clínicos e Diretrizes Terapêuticas do Ministério da Saúde ou, ainda, não havia CID nos documentos arquivados.

Dos vinte medicamentos mais pleiteados das demandas judiciais em Juiz de Fora (Tabela 3), dois são contemplados no Componente Básico (ácido valproico e carvedilol), três são contemplados em Protocolos Clínicos e Diretrizes Terapêuticas do Ministério da Saúde (rituximabe, clopidogrel e quetiapina), um consiste em antineoplásico incorporado pelo SUS (trastuzumabe) e um é contemplado no protocolo estadual de Doença Pulmonar Obstrutiva Crônica em Minas Gerais (brometo de tiotrópio).

Dos 13 medicamentos mais demandados e não disponibilizados pelo SUS, oito apresentam alternativa terapêutica disponível no SUS (oxcarbazepina, venlafaxina, cetuximabe, pregabalina, domperidona, insulina lispro, temozolomida e sorafenibe), ou seja, medicamentos de mesmo subgrupo farmacológico do sistema de classificação ATC, para a mesma indicação terapêutica.

Os cinco medicamentos mais demandados que não eram disponibilizados no SUS nem apresentavam alternativa terapêutica - ranibizumabe, metilfenidato, bevacizumabe, ácido ursodesoxicólico e rivaroxabana - apresentaram força de recomendaçáo classe IIa (recomendado na maioria dos casos) e grau de evidência categoria $B$ (evidência é baseada em dados derivados de meta-análise de estudos randomizados controlados com conclusóes conflitantes em relação às indicações e graus de resultados entre os estudos individuais) para as indicaçôes terapêuticas constantes na bula, com exceçáo do ácido ursodesoxicólico, que apresentou forma de recomendação classe I (uso recomendado). 
Dos 20 medicamentos mais pleiteados no período de estudo, 17 (85\%) foram demandados para indicaçóes terapêuticas diferentes das indicaçóes reconhecidas em bula (metilfenidato, oxcarbazepina, ácido valproico, bevacizumabe, brometo de tiotrópio, carvedilol, trastuzumabe, venlafaxina, pregabalina, rituximabe, domperidona, ácido ursodesoxicólico, insulina lispro, clopidogrel, sorafenibe, quetiapina e rivaroxabana).

\section{Discussão}

O estudo das demandas judiciais por medicamentos possibilitou a observação da polarização do município no fenômeno da judicialização da saúde na Zona da Mata Mineira. Nos processos em que Bicas, município limítrofe de Juiz de Fora, foi apontado como réu da ação, os juízes que deferiram os pedidos levaram em consideração a regionalização do SUS e a responsabilidade do município de Juiz de Fora no atendimento da demanda. Assim, os polos passivos foram retificados para fazer constar o município de Juiz de Fora no lugar do município de Bicas, mesmo não havendo acordo para a $\mathrm{AF}$ entre os dois municípios.

Em relação à origem das prescriçóes que motivaram as demandas, a prevalência de prescriçóes oriundas de serviços do SUS pode indicar a não adesão dos profissionais da rede pública às listas oficiais, ou ainda, atraso na incorporação de novos medicamentos às listas oficiais ${ }^{10,11}$. Por outro lado, vale ressaltar que muitos profissionais médicos que trabalham no sistema público de saúde também trabalham no sistema privado. Tendo em vista que seja exigida pelo juiz e recomendada pela Defensoria Pública do Estado de Minas Gerais a apresentação de receituário médico emitido por médico do SUS ou conveniado para dar início ao processo, pacientes oriundos do sistema privado podem ter suas receitas transcritas em receituários do sistema público.

As prescriçóes provenientes de hospitais universitários é um indicativo de demandas para medicamentos inovadores e/ou não incorporados ao SUS por se tratarem de centros de tratamento de alta complexidade. Além disso, os hospitais universitários situados no município prestam serviços de saúde de média e alta complexidade ao SUS municipal, mediante convênio com a prefeitura de Juiz de Fora. Dessa forma, 34\% (70 em 206) das prescriçóes oriundas de estabelecimentos privados de saúde são provenientes de instituições que prestam também serviços ao SUS.

Machado e colaboradores ${ }^{12}$ verificaram que apenas um profissional foi responsável pela emissão das prescriçóes que originaram 377 processos $(22,3 \%)$ em Minas Gerais. Os 217 médicos restantes atenderam 271 pacientes. Para esses mesmos autores, essa concentração de médicos pode sugerir a existência de interesses daqueles que comercializam medicamentos que vão além da proteção e recuperação da saúde do paciente e garantia de seu direito ao tratamento.

No que tange aos diagnósticos principais dos autores das açóes, as neoplasias também foram o principal diagnóstico identificado em Pernambuco e descrito por Marçal $^{13}$, em que $40,8 \%$ dos solicitantes apresentavam neoplasias como diagnóstico. As doenças do sistema nervoso e as neoplasias foram os principais diagnósticos encontrados por Lima ${ }^{14}$ no Rio de Janeiro, sendo responsáveis por $14,9 \%$ e $10,9 \%$ dos diagnósticos, respectivamente. As doenças do olho e anexos são apontadas por Marçal ${ }^{13}$ como terceiro capítulo diagnóstico mais prevalente $-11,3 \%$.

Dos 602 diagnósticos principais analisados, foi possível correlacionar $389 \mathrm{com}$ as características que dizem respeito ao estabelecimento de saúde que originou a prescrição médica. Foi possível identificar o estabelecimento de origem de $42,1 \%$ dos casos de neoplasias, $81 \%$ de doenças do olho e anexos, $63,4 \%$ de transtornos mentais e comportamentais, sendo que 89,6\% (43 em 48) dos casos de neoplasias e 70,4\% (57 em 81) dos casos de doenças do olho e anexos vieram de estabelecimentos privados, com predomínio da Associação dos Cegos em Juiz de Fora e 68,9\% (31 em 45) dos casos de transtornos mentais e comportamentais foram oriundos dos serviços públicos municipais de saúde.

Vale lembrar que a Secretaria Municipal de Saúde de Juiz de Fora firmou um contrato com a Agência de Cooperação Intermunicipal em Saúde Pé da Serra, a Fundação de Apoio ao Hospital Universitário e a Associação dos Cegos, na área de oftalmologia, no final de 2011. O contrato previa a realização de mais de 4.500 procedimentos por mês, entre consultas, exames e cirurgias, além do plantão 24 horas em oftalmologia pelo SUS, em Juiz de Fora. A garantia do atendimento de urgência e emergência em oftalmologia, inédito no SUS municipal, foi também a primeira iniciativa da macrorregião sudeste na especialidade ${ }^{15}$.

$\mathrm{O}$ aumento na capacidade de atendimento oftalmológico, desde a atenção primária até a urgência e emergência, pode ter contribuído para o aumento da frequência de diagnóstico das doenças do olho e anexos, com destaque para os transtornos da retina, já que tais doenças foram responsáveis por apenas $1,55 \%$ da amostra de açôes judiciais impetradas contra o município de Juiz de Fora em 2010, segundo dados de Felicíssimo ${ }^{16}$. $\mathrm{O}$ aumento do atendimento médico proporcionado pelo contrato de cooperação não previa, no entanto, a dispensaçáo de medicamentos, o que pode ter levado os pacientes diagnosticados com transtornos na retina a buscar o tratamento por via judicial. 
Nota-se que os diagnósticos principais que motivaram as demandas judiciais no período de estudo foram condiçóes crônicas, que correspondem a problemas de saúde que persistem e necessitam de cuidados permanentes, consequências naturais do processo das transiçôes demográfica e epidemiológica em curso no Brasil. A demanda judicial por medicamentos para o tratamento de condiçôes crônicas torna-se mais um indicativo da falha do sistema de saúde brasileiro em acompanhar o declínio dos problemas agudos e ascensão das condiçôes crônicas e da necessidade de implantação das redes de atenção à saúde, voltadas para a atenção às condiçóes crônicas, sem prescindir das condiçóes agudas.

Os medicamentos que atuam sobre o sistema nervoso e os antineoplásicos têm sido apontados por estudos em diversas regiōes do país como os mais pleiteados em açóes judiciais para o fornecimento de medicamentos ${ }^{3,12,17-19}$.

Apesar da impossibilidade de analisar a racionalidade do esquema terapêutico proposto, é possível constatar a coerência entre os diagnósticos prevalentes (neoplasias, doenças do olho e anexos, transtornos mentais e comportamentais) e os subgrupos terapêuticos dos medicamentos mais pleiteados (agentes antineoplásicos, oftalmológicos e os psicoanalépticos).

Estudos anteriores mostram que a maioria dos medicamentos sob litígio constavam em listas de financiamento público ${ }^{10,11,18}$. Estudos mais recentes mostram resultados semelhantes aos apresentados neste trabalho, indicando uma mudança no perfil dos processos, nos quais são requeridos, de forma mais proeminente, medicamentos não fornecidos pelo SUS ${ }^{12}$.

A predominância de demandas de medicamentos não disponibilizados pelo SUS pode sugerir diferentes situaçóes. Considerando o fato de que expressiva parte dos medicamentos foi solicitada por requerentes advindos de serviços públicos, os resultados encontrados podem refletir que essas unidades não estão utilizando os medicamentos padronizados pelo sistema de saúde. Por outro lado, pode dar sinais de que as necessidades terapêuticas dos usuários não estão sendo satisfeitas com as alternativas terapêuticas do SUS ou ainda de que a publicidade de medicamentos lançados no mercado farmacêutico brasileiro e não disponibilizados pelo SUS tem interferido na escolha dos prescritores.

Em outros locais de estudo, os medicamentos previstos no componente básico da assistência farmacêutica aparecem em proporções semelhantes às encontradas: 8,4\% em Pernambuco e 10,9\% em Minas Gerais, chegando a 24,1\% no Rio de Janeiro. A proporção de medicamentos do elenco do Componente Estratégico da AF encontrada foi ligeiramente maior no Rio de Janeiro $(1,9 \%)$ e em Pernambuco (2,6\%), alcançando 3,5\% no estado de Minas Gerais ${ }^{12-14}$.
Dos sete medicamentos contemplados no Componente Estratégico, quatro possibilitaram a correlação com o estabelecimento de origem da prescrição: dois foram prescritos em um hospital geral privado sem fins lucrativos, um em hospital universitário e um em Unidade Básica de Saúde.

Ao contrário do que foi encontrado neste estudo (maior busca pelos medicamentos básicos), os medicamentos previstos no Componente Especializado da AF foram os mais solicitados por via judicial no Rio de Janeiro (19,4\%), em Minas Gerais (25\%), em Pernambuco $(10,8 \%)$ e no Paraná $(26,4 \%)^{13,20-22}$. Por outro lado, assim como encontrado aqui, Lima ${ }^{14}$ identificou maior proporção de medicamentos do Componente Básico $(24,1 \%)$ do que de medicamentos do Componente Especializado (17,2\%).

Estudos anteriores mostram que a maioria dos medicamentos sob litígio eram medicamentos constantes em listas de financiamento público ${ }^{10,11,18,23}$. Já trabalhos mais recentes mostram resultados semelhantes aos apresentados aqui, indicando uma mudança no perfil dos processos, nos quais são requeridos, de forma mais proeminente, medicamentos não fornecidos pelo SUS ${ }^{12,24,25}$.

A análise das demandas judiciais por medicamentos no biênio estudado em Juiz de Fora possibilitou identificar quatro categorias de medicamentos, a saber: 1) medicamentos sem registro na Anvisa; 2) medicamentos incorporados pelo SUS; 3) medicamentos nâo disponibilizados pelo SUS; e 4) medicamentos disponibilizados ou não pelo SUS para uso off label.

Os medicamentos sem registro na Anvisa fazem parte da categoria de medicamentos identificada menos expressiva $(0,7 \%)$, contudo, não menos importante do ponto de vista da eficácia e segurança do tratamento medicamentoso, já que a soluçâo oral de captopril a 2 $\mathrm{mg} / \mathrm{mL}$ foi prescrita para o tratamento de vômitos excessivos na gravidez (CID O21.0), mesmo sendo contraindicado durante a gravidez devido ao risco de causar danos e até a morte do feto ${ }^{26}$.

A segunda categoria, formada pelos medicamentos incorporados pelo SUS, divide-se em duas subcategorias: a) medicamentos contemplados nas listas de financiamento e nos Protocolos Clínicos e Diretrizes Terapêuticas; e b) medicamentos do Componente Estratégico de AF demandados para situaçóes clínicas diferentes das previstas nos Protocolos Clínicos.

A demanda de medicamentos contemplados nas listas de financiamento público sugere falhas na gestão da AF pública, ao passo que a diminuição da proporção de demanda desses medicamentos sugere a melhoria da qualidade da prestaçáo dos serviços de AF no SUS. Por outro lado, a pequena proporção de medicamentos fornecidos pelo SUS pode indicar o deferimento pelo juiz 
de todos os medicamentos presentes no receituário que respaldou a ação, mesmo não sendo objeto do pleito.

Já os medicamentos da subcategoria "medicamentos do Componente Estratégico demandados para situaçóes clínicas diferentes das previstas nos Protocolos Clínicos e Diretrizes Terapêuticas" atingiram 10,5\% daqueles pleiteados no período de estudo, podendo indicar falha na atualização dos protocolos ou das indicaçôes registradas em bula ou mesmo prescrição para uso off label sem grau de evidência.

Por fim, a quarta categoria - medicamentos incorporados ou não no SUS, demandados para o tratamento de indicações terapêuticas diferentes das indicaçôes reconhecidas em bula, mostrou-se expressiva entre os 20 medicamentos mais pleiteados neste estudo. A utilização de um medicamento já registrado para uma indicação não regulamentada é uma prática bastante difundida. Embora a promoção e a publicidade de medicamentos para uso off label seja proibida no Brasil - de acordo com a RDC no 96, de 17 de dezembro de 2008 -, o uso off label de medicamentos náo é ilegal e pode ser clinicamente apropriado em circunstâncias em que os potenciais benefícios superam os riscos. Por outro lado, o uso de medicamentos no tratamento de situaçóes clínicas para as quais não foram registrados pode significar riscos à saúde, especialmente quando não foram realizados ensaios clínicos a fim de confirmar a eficácia e a segurança do medicamento para determinado uso $^{3,27}$.

\section{Conclusões}

Por ser um município polo regional de saúde, que atende à demanda de atendimento de média e alta complexidade da população residente em cidades próximas da fronteira interestadual, Juiz de Fora prevê uma oferta de serviços de saúde a uma populaçáo adscrita de acordo com o Plano Diretor de Regionalização de Minas Gerais. Além disso, o município é também impelido a estender seu atendimento a populaçóes fora do planejamento original da AF, sem ressarcimento dos custos pelos outros municípios. Assim, o expressivo número de açóes judiciais sem informação sobre o domicílio do autor da ação $(41,6 \%)$ torna-se um importante tópico a ser investigado, com vistas a identificar se os autores dessas açôes são residentes de Juiz de Fora, de municípios adscritos à micro e/ou macrorregiấo de saúde de Juiz de Fora ou de municípios à margem do planejamento das ações de saúde. Com a obtenção desses dados, cria-se a possibilidade de retificaçấo do polo passivo da açáo judicial, considerando a regionalização do SUS e a responsabilidade dos municípios polos micro e macrorregionais.
Em relação às prescriçóes médicas que respaldaram a ação judicial, o predomínio de prescriçóes advindas de estabelecimentos públicos de saúde e de estabelecimentos privados que prestam serviços ao SUS indica que a própria rede de serviços do SUS sobrecarrega o sistema com as demandas judiciais. Portanto, a interação do gestor municipal de saúde com os prescritores vinculados aos prestadores de serviços do SUS deve ocorrer de modo a alcançar o entendimento entre os atores envolvidos e a adesão de prescritores às normas para prescriçôes de medicamentos no âmbito do SUS.

Já quanto ao fornecimento público dos medicamentos pleiteados, foi possível observar o predomínio de açóes judiciais que demandam medicamentos não fornecidos pelo SUS, assim como ocorre em diversas regiōes do Brasil. Esse panorama pode refletir a não utilização dos medicamentos padronizados pelo sistema de saúde e a não satisfação das necessidades terapêuticas dos pacientes com as alternativas disponíveis no SUS.

Além disso, torna-se importante o aprofundamento do estudo sobre o expressivo uso off label identificado no estudo com vistas à garantia da eficácia e segurança dos usuários do sistema de saúde.

Portanto, é imprescindível que sejam continuamente investigadas as particularidades das demandas judiciais por medicamentos em municípios polos como Juiz de Fora a fim de que o fenômeno da judicialização seja considerado no planejamento da saúde, na articulação interfederativa e na assistência à saúde.

\section{Referências}

1. Brasil - Diário Oficial da República Federativa do Brasil. Lei no 8.080 , de 19 de setembro de 1990. Dispóe sobre as condiçôes para promoção, proteção e recuperação da saúde, a organização e o funcionamento dos serviços e da outras providências. Brasília, DF; 1990.

2. Brasil. Ministério da Saúde. Portaria no 2.203, de 5 de novembro de 1996. Dispóe sobre a Norma Operacional Básica do SUS 01/96. Brasília, DF; 1996.

3. Pepe VLE, Figueiredo TA, Simas L, Osório-de-Castro CGS, Ventura M. A Judicialização da Saúde e os novos desafios da gestão da assistência farmacêutica. Ciênc Saúde Coletiva. 2010;15(5):2405-14.

4. Figueiredo TA, Pepe VLE, Osório-de-Castro CGS. Um enfoque sanitário sobre a demanda judicial de medicamentos. Physis. 2010;20(1):101-18.

5. Brasil - Diário Oficial da República Federativa do Brasil. Decreto no 7.508, de 28 de junho de 2011. Regulamenta a Lei $n^{\circ} 8.080$, de 19 de setembro de 1990, para dispor sobre a organização do Sistema Único de Saúde - SUS, o planejamento da saúde, a assistência à saúde e a articulação interfederativa, e dá outras providências. Brasília, DF; 2011. 
6. Instituto Brasileiro de Geografia e Estatística. IBGE cidades: 2014 [citado em 2014 jun 24]. Disponível em: http://www.cidades.ibge.gov.br/xtras/perfil. php?lang=\&codmun=313670.

7. Malachias I, Leles FAG, Pinto MAS. Plano Diretor de Regionalização da Saúde de Minas Gerais. Belo Horizonte: Secretaria de Estado de Saúde de Minas Gerais; 2011.

8. Pepe VLE, organizadora. Manual Indicadores de avaliação e monitoramento das demandas judiciais de medicamentos. Rio de Janeiro: Fiocruz, Ensp; 2011.

9. Machado MAA. Acesso a medicamentos via poder judiciário no estado de Minas Gerais [dissertação]. Belo Horizonte: Faculdade de Farmácia, Universidade Federal de Minas Gerais; 2010.

10. Messeder AM, Osório-de-Castro CGS, Luzia VL. Mandados judiciais como ferramentas para garantia do acesso a medicamentos no setor público: a experiência do estado do Rio de Janeiro. Cad Saúde Pública. 2005;21(2):525-34.

11. Vieira FS, Zucchi P. Distorçôes causadas pelas ações judiciais à política de medicamentos no Brasil. Rev Saúde Pública. 2007;41(2):214-22.

12. Machado MAA, Acurcio FA, Brandão CMR, Faleiros DR, Guerra Jr AA, Cherchiglia ML, et al. Judicialização do acesso a medicamentos no estado de Minas Gerais, Brasil. Rev Saúde Pública. 2011;45(3):590-8.

13. Marçal KKS. A judicialização da Assistência Farmacêutica: o caso Pernambuco em 2009 e 2010 [dissertação]. Recife: Centro de Pesquisas Aggeu Magalhães, Fundação Oswaldo Cruz; 2012.

14. Lima GS. Demanda judicial de medicamentos e uso de indicadores de avaliaçáo e monitoramento no estado do Rio de Janeiro [dissertação]. Rio de Janeiro: Escola Nacional de Saúde Pública Sérgio Arouca, Fundação Oswaldo Cruz; 2012.

15. Miranda C. Centro Oftamológico $24 \mathrm{~h}$ é inaugurado em Juiz de Fora. Diário Regional [Internet]. 2011 [citado em 2014 set 24]. Disponível em: http://www.uff.br/ hu/2011/12/16/16-de-dezembro-de-2011/

16. Felicíssimo JM. Judicialização de saúde para o fornecimento de medicamentos: análise das açôes impetradas contra o município de Juiz de Fora, MG em 2010 [trabalho de conclusão de curso]. Juiz de Fora: Faculdade de Medicina, Universidade Federal de Juiz de Fora; 2012.
17. Boing AC, Bloemer NS, Roesler C, Fernandes S. A judicializaçáo do acesso aos medicamentos em Santa Catarina: um desafio para a gestáo do sistema de saúde. R Dir Sanit. 2013;14(1):82-97.

18. Romero LC. Judicialização das políticas de assistência farmacêutica: o caso do distrito federal. Brasília, DF: Consultoria Legislativa do Senado Federal; 2008.

19. Stamford A, Cavalcanti M. Decisóes judiciais sobre acesso aos medicamentos em Pernambuco. Rev Saúde Pública. 2012;46(5):791-9.

20. Macedo EI, Lopes LC, Barberato-Filho S. Análise técnica para a tomada de decisão do fornecimento de medicamentos pela via judicial. Rev Saúde Pública. 2011;45(4):706-13.

21. Figueiredo TA. Análise dos medicamentos fornecidos por mandado judicial na comarca do Rio de Janeiro: a aplicação de evidências científicas no processo de tomada de decisão [dissertação]. Rio de Janeiro: Escola Nacional de Saúde Pública Sérgio Arouca, Fundação Oswaldo Cruz; 2010.

22. Pereira JG. Demandas judiciais de medicamentos no estado do Paraná: aplicação de indicadores de avaliação e monitoramento e qualidade de vida relacionada à saúde dos usuários [tese]. Rio de Janeiro: Escola Nacional de Saúde Pública Sérgio Arouca, Fundação Oswaldo Cruz; 2013.

23. Sant'ana JMB. Essencialidade e assistência farmacêutica: um estudo exploratório das demandas judiciais individuais para acesso a medicamentos no estado do Rio de Janeiro [dissertação]. Rio de Janeiro: Escola Nacional de Saúde Pública Sérgio Arouca, Fundação Oswaldo Cruz; 2009.

24. Barcelos PC. Perfil de demandas judiciais de medicamentos da Secretaria de Estado da Saúde do Espírito Santo: um estudo exploratório [dissertação]. Rio de Janeiro: Instituto de Medicina Social, Universidade Estadual do Rio de Janeiro; 2010.

25. Rosein JG. Análise do perfil das solicitaçóes de medicamentos por demanda judicial no Estado de Santa Catarina no período de 2005 a 2008 [dissertação]. Florianópolis: Universidade Federal de Santa Catarina; 2010.

26. Captosen: captopril. [Bula]. Lagoa da Prata: Pharlab Indústria Farmacêutica S.A.; 2014.

27. Ramos KA, Ferreira ASD. Análise da demanda de medicamentos para uso off label por meio de açóes judiciais na Secretaria de Estado de Saúde de Minas Gerais. R Dir Sanit. 2013;14(1):98-121.

\section{Como citar este artigo:}

Munck AKR, Lupatini EO, Pereira RMC, Vieira RCPA. Demandas judiciais de medicamentos em um município polo de Minas Gerais. Rev. Aten. Saúde. 2016;14(50):5-12. 